\title{
RESTORING ARTIFACT-FREE MICROSCOPY IMAGE SEQUENCES
}

\author{
Zhaozheng Yin Takeo Kanade \\ Robotics Institute \\ Carnegie Mellon University \\ 5000 Forbes Ave, Pittsburgh, PA 15213, USA
}

\begin{abstract}
Phase contrast and differential interference contrast, which are used to capture microscopy images of living cells, contain a few artifacts such as halo and shadow-cast effect. Removing these artifacts from microscopy images facilitates automated microscopy image analysis, such as the cell segmentation that is a critical step in cell tracking systems. We propose a restoration algorithm based on the microscopy imaging model and consider temporal consistency when restoring time-lapse microscopy image sequences. The artifact-free microscopy images are restored by minimizing a regularized quadratic cost function that is adaptable to input image properties. Our method achieves high segmentation accuracy and low computational cost compared to the previous methods.
\end{abstract}

Index Terms - Microscopy image analysis, image restoration, phase contrast, differential interference contrast

\section{INTRODUCTION}

The demand of visualizing transparent cells without altering the specimen themselves motivated microscopists to develope several successful microscopy techniques in the past century. Based on the observation that cells change the phase of incident wavefronts due to their thickness and refractive index different from the surrounding medium, phase contrast and differential interference contrast (DIC) microscopy techniques were invented to convert the minute phase variations to intensity changes for human observation ([5], Chapter 7 and 10). As shown in Fig. 1(a), when imaged by positive phase contrast optics, cells appear darker than the surrounding medium. When imaged by a DIC microscope, cells have the 3D-like topographical appearance (Fig. 1(b)).

However, several inherent artifacts can be observed in these microscopy images. There are bright halos around cell membranes in the phase contrast image (Fig. 1(a)), thus the observed pixel intensities around halo regions do not directly reflect the specimen's properties. There is a pseudo 3D shading effect in the DIC image (Fig. 1(b)) where regions with increasing optical path difference appear brighter than those with decreasing optical path difference. This shadow-cast effect only indicates the sign and slope orientation of optical

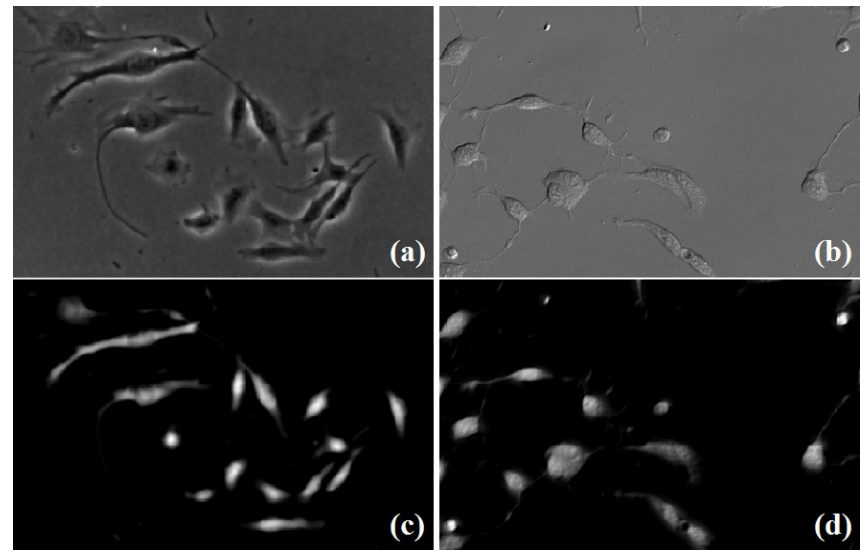

Fig. 1. Restoring artifact-free microscopy images to facilitate cell segmentation. (a) Phase contrast image with halo artifacts; (b) DIC image with pseudo 3D shadow-cast effect; (3) Restored phase contrast image; (4) Restored DIC image.

path gradients and it does not indicate the actual topographical structures of cells. These artifacts have hindered the process of automated microscopy image analysis, such as the cell segmentation that is a critical step in automated cell tracking systems $[3,6]$.

Recently, we proposed a method to restore artifact-free microscopy images that are suitable for segmentation by direct thresholding $[4,9]$. Our method minimizes a regularized quadratic cost function defined by the imaging models derived from the optical principles of phase contrast and DIC microscopes. In this paper, we enhance the previous method in four folds: (1) extend the algorithm to restore microscopy video sequences by considering temporal consistency; (2) allow for tuning the regularization terms in the cost function according to observed image properties; (3) devise a better initialization method by using a look-up table built from input image properties so that the restoration is more adaptable; (4) largely reduce the computational cost without sacrificing the algorithm's accuracy. Fig. 1(c) and (d) show the results of our restoration method corresponding to Fig. 1(a) and (b), respectively, which are artifact-free and amenable to the basic thresholding method for segmenting cells from the background. 


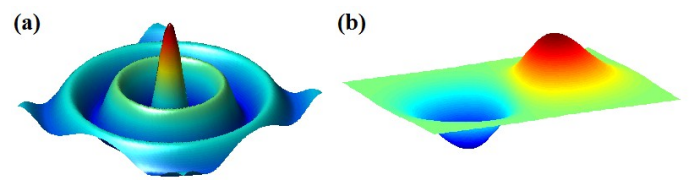

Fig. 2. Convolutional kernels used in our linear imaging model. (a) Obscured airy kernel in the phase contrast imaging; (b) Difference-of-Gaussian kernel in the DIC imaging.

\section{RESTORATION ALGORITHM ON IMAGE SEQUENCES}

The artifacts included in Fig. 1(a) and (b) can be well explained by the optical properties of the microscopy imaging formation process. In $[4,9]$, we have derived a linear microscopy imaging model

$$
\mathbf{g}=\mathbf{H f}
$$

where $\mathbf{g}$ is a vectorized observed image, $\mathbf{H}$ is a matrix defined by the imaging optics, and $\mathbf{f}$ is the vectorized artifact-free image to be restored. In the phase contrast imaging, $\mathbf{H}$ corresponds to an obscured airy kernel (Fig. 2(a), [9]), and in the DIC imaging, $\mathbf{H}$ corresponds to a directional Difference-ofGaussian kernel (Fig. 2(b), [4]).

Directly inversing $\mathbf{H}$ to restore $\mathbf{f}$ from $\mathbf{g}$ in Eq. 1 is known to be highly noise-prone. Instead, given an observed sequence of $N$ images, $\left\{\mathbf{g}^{(t)}\right\}_{t=1, \cdots, N}$, we restore the artifact-free sequence, $\left\{\mathbf{f}^{(t)}\right\}_{t=1, \cdots, N}$, by minimizing the following cost function $^{1}$

$$
\begin{aligned}
\mathbf{O}(\mathbf{f})= & \|\mathbf{H} \mathbf{f}-\mathbf{g}\|_{2}^{2}+\omega_{s} \mathbf{f}^{T} \mathbf{L} \mathbf{f}+\omega_{r}\|\mathbf{\Lambda} \mathbf{f}\|_{1}+ \\
& \omega_{t}\left(\mathbf{f}-\mathbf{f}^{(t)}\right)^{T} \mathbf{\Sigma}\left(\mathbf{f}-\mathbf{f}^{(t)}\right)
\end{aligned}
$$

where $\mathbf{L}$ is a Laplacian matrix defining the similarity between spatial pixel neighbors, $\boldsymbol{\Lambda}$ is a positive diagonal matrix defining the $l_{1}$ sparseness regularization, and $\boldsymbol{\Sigma}$ is a matrix defining the similarity between temporal pixel neighbors. $\mathbf{L}, \boldsymbol{\Lambda}$ and $\boldsymbol{\Sigma}$ are explained in Section 3. The temporal consistency term is dropped when restoring the first image of a sequence. The weighting coefficients on different regularization terms $\left(\omega_{s}\right.$, $\omega_{r}$ and $\omega_{t}$ ) are to be learned by grid-search [4, 9]. Because of the $l_{1}$ sparseness term, there is no closed-form solution to $\mathbf{O}(\mathbf{f})$. Instead, we constrain the restored $\mathbf{f}$ to have nonnegative values and convert Eq. 2 to the following optimization problem

$$
\mathbf{O}(\mathbf{f})=\mathbf{f}^{T} \mathbf{Q f}+2 \mathbf{b}^{T} \mathbf{f}+c \quad \text { s.t. } \mathbf{f} \geq 0
$$

where

$$
\begin{aligned}
\mathbf{Q} & =\mathbf{H}^{T} \mathbf{H}+\omega_{s} \mathbf{L}+\omega_{t} \boldsymbol{\Sigma} \\
\mathbf{b} & =-\mathbf{H}^{T} \mathbf{g}-\omega_{t} \boldsymbol{\Sigma}^{T} \mathbf{f}^{(t)}+\omega_{r} \operatorname{diag}(\boldsymbol{\Lambda}) / 2 \\
c & =\mathbf{g}^{T} \mathbf{g}+\omega_{t} \mathbf{f}^{(\mathbf{t})^{T}} \mathbf{f}^{(\mathbf{t})} .
\end{aligned}
$$

\footnotetext{
${ }^{1}$ To simplify notation, we denote $\mathbf{f}$ as the image to be restored at time $t+1, \mathbf{g}$ as the image observed at time $t+1$, and $\mathbf{f}^{(t)}$ as the restored image at time $t$.
}

We propose an iterative algorithm to solve $\mathbf{f}$ in Eq. 3 by non-negative multiplicative updating (Eq. 8, [7]). Reweighting (Eq. 9, [2]) is an option to accelerate the convergence process.

\section{Algorithm I: restoring artifact-free microscopy images}

Initialize $\mathbf{f}=\mathbf{f}^{\text {init }}$ and $\boldsymbol{\Lambda}=\boldsymbol{\Lambda}^{\text {init }}$.

Repeat the following steps for all pixel $j$

$$
\begin{aligned}
\mathbf{b} & =-\mathbf{H}^{T} \mathbf{g}-\omega_{t} \boldsymbol{\Sigma}^{T} \mathbf{f}^{(t)}+\omega_{r} \operatorname{diag}(\boldsymbol{\Lambda}) / 2 \\
\mathbf{f}_{j} & \leftarrow \mathbf{f}_{j} \frac{-\mathbf{b}_{j}+\sqrt{\mathbf{b}_{j}^{2}+4\left(\mathbf{Q}^{+} \mathbf{f}\right)_{j}\left(\mathbf{Q}^{-} \mathbf{f}\right)_{j}}}{2\left(\mathbf{Q}^{+} \mathbf{f}\right)_{j}} \\
\boldsymbol{\Lambda}_{j j} & \leftarrow \frac{\boldsymbol{\Lambda}_{j j}^{i n i t}}{\mathbf{f}_{j}+\epsilon}
\end{aligned}
$$

Until convergence.

where $\epsilon$ is a small constant to avoid divide-by-zero, $\mathbf{Q}^{+}$and $\mathbf{Q}^{-}$store the positive and negative elements of $\mathbf{Q} . \boldsymbol{\Lambda}^{\text {init }}$ is the sparse regularization defined by input image properties (Section 3) and $\mathbf{f}^{\text {init }}$ is the initialization obtained from a lookup table (Section 4).

\section{REGULARIZATIONS DEFINED BY IMAGE PROPERTIES}

In a microscopy video sequence, neighboring pixels are linked in both spatial and temporal domain, we define the similarity between spatial neighbors as

$$
\mathbf{W}_{m n}=e^{-\left(\mathbf{g}_{m}-\mathbf{g}_{n}\right)^{2} / \sigma_{1}}
$$

where $\mathbf{g}_{m}$ and $\mathbf{g}_{n}$ denote intensities of neighboring pixels $m$ and $n$, and $\sigma_{1}$ is the mean of all possible $\left(\mathbf{g}_{m}-\mathbf{g}_{n}\right)^{2}$, $s$ in the image. The spatial smoothness regularization is defined as

$$
\mathbf{f}^{T} \mathbf{L f}=\sum_{m, n \in \Omega(m)} \mathbf{W}_{m n}\left(\mathbf{f}_{m}-\mathbf{f}_{n}\right)^{2}
$$

where $\Omega(m)$ denote the spatial 8-connected neighborhood of pixel $m$. Explicitly, we compute $\mathbf{L}=\mathbf{D}-\mathbf{W}$ where $\mathbf{D}_{m m}=$ $\sum_{n} \mathbf{W}_{m n}$.

Similarly, we define the similarity between two neighboring pixels in the temporal domain as

$$
\boldsymbol{\Sigma}_{m m}=e^{-\left(\mathbf{g}_{m}-\mathbf{g}_{m}^{(t)}\right)^{2} / \sigma_{2}}
$$

where $\mathbf{g}_{m}^{(t)}$ denotes the intensity of pixel $m$ in the previous time instant, and $\sigma_{2}$ is the mean of all possible $\left(\mathbf{g}_{m}-\mathbf{g}_{m}^{(t)}\right)^{2}$, s between two consecutive images. Each pixel in the current image can be connected to nine temporal neighbors in the previous frame. The regularization terms on spatial and temporal smoothness enforce neighboring pixels with similar observed values to have similar values in the restored image.

The sparsity regularization in Eq. 2 enforces most pixels of the restored artifact-free image to be close to zero. Ideally, 

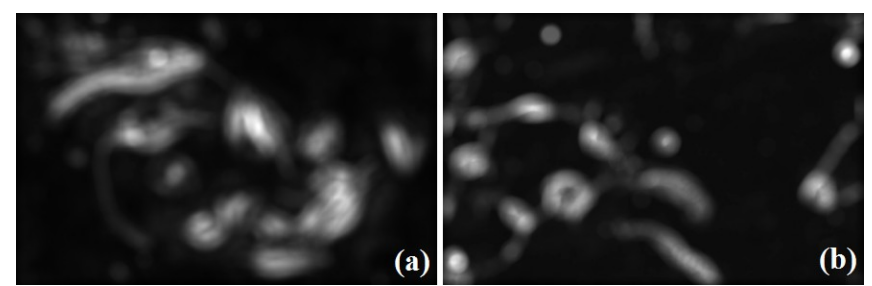

Fig. 3. Sparseness regularization by spatial image frequency analysis, corresponding to Fig. 1(a) and (b), respectively.

all the background pixels have zero values while cell pixels have positive values. In $[4,9]$, the sparseness is implemented by initializing $\boldsymbol{\Lambda}$ as a constant at the beginning (i.e. $\boldsymbol{\Lambda}^{\text {init }}=$ I) and re-weight it in the iterative process by

$$
\Lambda_{j j} \leftarrow \frac{1}{\mathbf{f}_{j}+\epsilon} .
$$

Rather than blindly enforce the sparseness over all pixels, we tune the sparsity regularization term according to the spatial image frequency. First, we apply the 2D Fourier transform, $\mathcal{F}$, on image $\mathbf{g}$

$$
\mathbf{G}=\mathcal{F}(\mathbf{g}) .
$$

$\mathbf{G}$ is an image with complex values where the magnitude, $\mathcal{A}(\mathbf{G})$, tells how much of a certain frequency component appears, and the phase, $\mathcal{P}(\mathbf{G})$, indicates where the frequency component is in the image. Then, we apply a high pass filter on the frequency magnitude to get $\mathcal{A}^{\prime}(\mathbf{G})$ (i.e. set all frequency components below a cutoff frequency to zero.) Finally, we transform back to the spatial domain by inverse $2 \mathrm{D}$ Fourier transform

$$
\mathbf{g}^{\prime}=\mathcal{F}^{-1}\left(\mathcal{A}^{\prime}(\mathbf{G}) e^{i \mathcal{P}(\mathbf{G})}\right) .
$$

Fig. 3(a) and (b) shows the high pass filtering on Fig. 1(a) and (b), respectively, where bright regions represent high frequency components corresponding to possible cell pixels. Thus, we define the initial sparsity matrix by

$$
\mathbf{\Lambda}^{i n i t}=\operatorname{diag}\left(e^{-\mathbf{g}^{\prime}}\right)
$$

and also use it in the re-weighting step (Eq. 9). Using this sparsity regularization in the cost function, the lower frequency regions corresponding to background pixels are penalized more than higher frequency components corresponding to cell pixels.

\section{INITIALIZATION INFERRED FROM A LOOK-UP TABLE}

To solve the nonnegative quadratic problem in Eq. 3, a good initialization close to the optimal solution will let the iterative process converge fast. Rather than using the constant initialization as in $[4,9]$, we build a look-up table to infer a better initialization. Given a training sequence, g's, we run Algorithm I with $\mathbf{f}^{\text {init }}=\mathbf{1}$ and restore a sequence of $\mathbf{f}$ 's. Then, we calculate a histogram $h\left(\mathbf{g}_{j},|\dot{\mathbf{g}}|_{j}, \mathbf{f}_{j}\right)$ over every pixel based

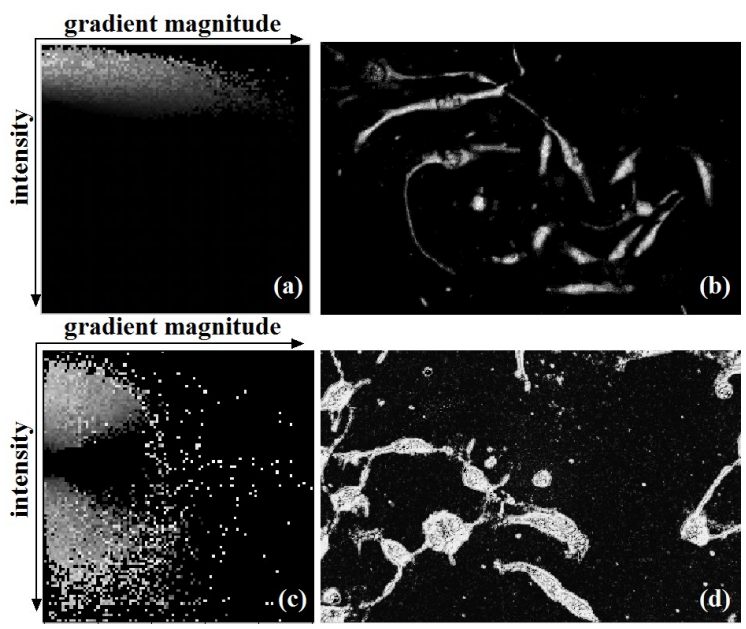

Fig. 4. Initialization for the iterative algorithm. (a) A look-up table for phase contrast images; (b) The inferred initialization for Fig. 1(a); (c) A look-up table for DIC images; (d) The inferred initialization for Fig. 1(b).

on the observed image intensity $\mathbf{g}_{j}$, image gradient magnitude $|\dot{\mathbf{g}}|_{j}$ and restored $\mathbf{f}_{j}$ value. The look-up table is computed as

$$
\operatorname{Tbl}\left(\mathbf{g}_{j},|\dot{\mathbf{g}}|_{j}\right)=\underset{\mathbf{f}_{j}}{\arg \max } h\left(\mathbf{g}_{j},|\dot{\mathbf{g}}|_{j}, \mathbf{f}_{j}\right) .
$$

Fig. 4(a) shows the computed look-up table from a training phase contrast sequence where the positive $f$ values (scattered bright regions) concentrate on entries with low intensity observations and a wide range of gradient observations. This phenomenon is commonly observed from any positive phase contrast image where cell pixels appear dark and have various local gradients. Based on this look-up table, for every pixel $j$ in an image $\mathbf{g}$ to be restored, we infer the initialization as $\mathbf{f}_{j}^{\text {init }}=\operatorname{Tbl}\left(\mathbf{g}_{j},|\dot{\mathbf{g}}|_{j}\right)$. Note, if $\mathbf{f}_{j}^{\text {init }}$ is zero, we set it to a small positive constant to avoid being stuck at zero in Eq. 8 . The inferred initialization for Fig. 1(a) is shown in Fig. 4(b) that is close to the optimal solution except some isolated cell regions and background noise.

Fig. 4(c) shows the look-up table from a training DIC sequence where most positive $\mathbf{f}$ values are from entries corresponding to either high or low intensity observations and a wide range of gradient observations. This look-up table is coincident with the common observations in DIC images: the bright and dark pixels correspond to increasing or decreasing optical path lengths showing the pseudo 3D shading effect. Figure 4(d) shows the inferred initialization for Figure 1(b).

Basically, the loop-up table is equivalent to solving a single global maximum-a-posterior problem

$$
\underset{\mathbf{f}_{j}}{\arg \max } p\left(\mathbf{f}_{j} \mid\left(\mathbf{g}_{j},|\dot{\mathbf{g}}|_{j}\right)\right) \text {. }
$$

Other soft-segmentation methods such as a bag of local Bayesian classification [8] can be adopted here to provide an initialization for our iterative restoration algorithm. 


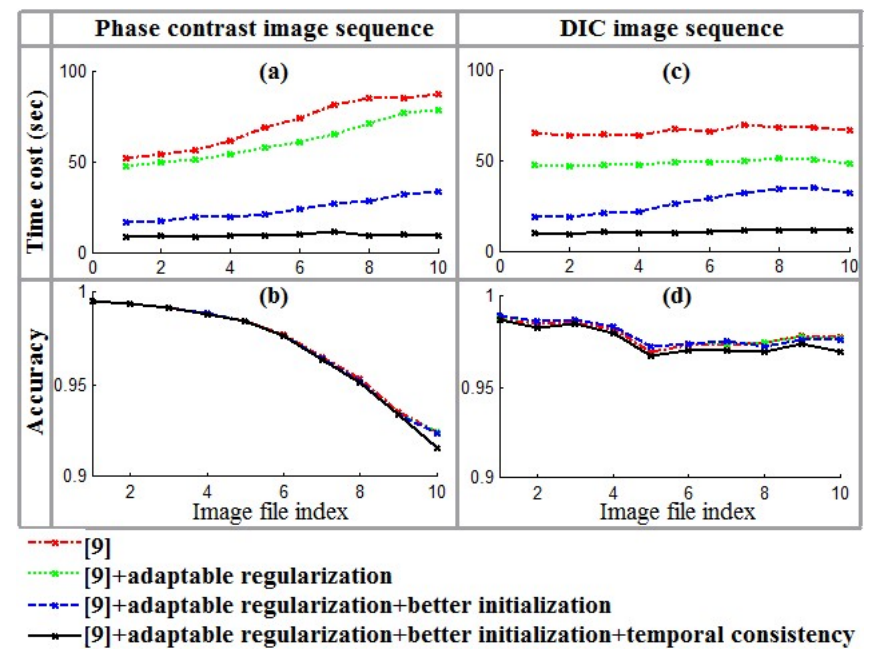

Fig. 5. Comparing the time cost and accuracy of different algorithm implementations with or without image-adapted regularization, initialization or temporal consistency.

\section{RESULTS}

To evaluate our algorithm's performance, we recorded 1000 phase contrast images over four days and another set of 1000 DIC images, then we manually label all cell masks every 100th image as ground truth. We adopt the evaluation metrics in [9] to compare four different algorithm implementations: (1) original restoration algorithm in [9]; (2) revised [9] with regularization terms adapted to image properties; (3) revised [9] with adaptable regularization terms and a better initialization from a look-up table; (4) revised [9] with all our proposed techniques.

The phase contrast image dataset includes a growing number of stem cells (from 20+ to 500+, left column of Fig. 6). As the cell number increases, the image has more nonnegative $\mathbf{f}$ values (cell pixels) and this causes more element-wise operations in Eq. 8, thus the time cost increases from the beginning to the end of this sequence, as shown in Fig. 5(a). Meanwhile, the restoration accuracy decreases a little bit in Fig. 5(b) because a large number of cells cultured in a dish may occlude each other and blur the halo effect. The number of cells in the DIC image sequence does not change a lot over time (top row of Fig. 7), thus the time cost and accuracy maintain relatively stable from the beginning to the end of this sequence, as shown in Fig. 5(c) and (d). From Fig. 5(a) and (c), we found that the implementation with all proposed techniques has the least time cost. From Fig. 5(b) and (d), we observe that the four algorithm implementations have competitive accuracies compared to each other. The reason is because our iterative algorithm to solve the nonnegative quadratic optimization problem in Eq. 3 is guaranteed to converge to a unique global optimum [7].

Overall, our proposed method achieves high restoration accuracy with lower computational cost compared to the previous method, which is suitable for online large-scale cell tracking experiments. Fig. 6 and 7 show some detailed restoration results by our algorithm where cell pixels have positive values while background pixels have uniform zero values, which favors the basic segmentation by thresholding. The overlapped cells can be handled by the algorithm in [1].

\section{CONCLUSION}

The artifacts in phase contrast and DIC microscopy hinder the automated microscopy image analysis such as cell segmentation that is a critical step in cell tracking systems. We propose a restoration algorithm based on the microscopy imaging model and consider temporal consistency when restoring time-lapse microscopy image sequences. The restoration is accomplished by minimizing a regularized quadratic cost function iteratively. The regularization terms are adapted to input image properties. To accelerate the iterative process, we infer an initialization close to the optimal solution by using a look-up table built from training images. The restored artifact-free images are amenable to cell segmentation by the basic thresholding method. Compared with the previous method [9], our proposed approach achieves high segmentation accuracy with lower computational cost, which can greatly facilitate online large-scale cell image segmentation and cell tracking applications.

\section{REFERENCES}

[1] R. Bise, K. Li, S. Eom and T. Kanade, "Reliably Tracking Partially Overlapping Neural Stem Cells in DIC Microscopy Image Sequences," Proceedings of MICCAI workshop on OPTMHisE, 2009.

[2] E. Candes, M. Wakin and S. Boyd, "Enhancing Sparsity by Reweighted 11 Minimization," The Journal of Fourier Analysis and Applications, 14(5): 877-905, 2008.

[3] K. Li, E.D. Miller, M. Chen, T. Kanade, L. Weiss and P. Campbell, "Cell Population Tracking and Lineage Construction with Spatiotemporal Context," Medical Image Analysis, 12(5):546$566,2008$.

[4] K. Li and T. Kanade, "Nonnegative Mixed-Norm Preconditioning for Microscopy Image Segmentation," Proceedings of IPMI, 2009.

[5] D. Murphy, "Fundamentals of Light Microscopy and Electronic Imaging," Wiley, 2001.

[6] J. Rittscher, "Characterization of Biological Processes through Automated Image Analysis," Annual Review of Biomedical Engineering, 12: 315-344, 2010.

[7] F. Sha, Y. Park and L. Saul, "Multiplicative Updates for Nonnegative Quadratic Programming," Neural Computation, 19(8):2004-2031, 2007.

[8] Z. Yin, R. Bise, T. Kanade and M. Chen, "Cell Segmentation in Microscopy Imagery Using a Bag of Local Bayesian Classifiers," Proceedings of ISBI, 2010.

[9] Z. Yin, K. Li, T. Kanade and M. Chen, "Understanding the Optics to Aid Microscopy Image Segmentation," Proceedings of MICCAI, 2010. 


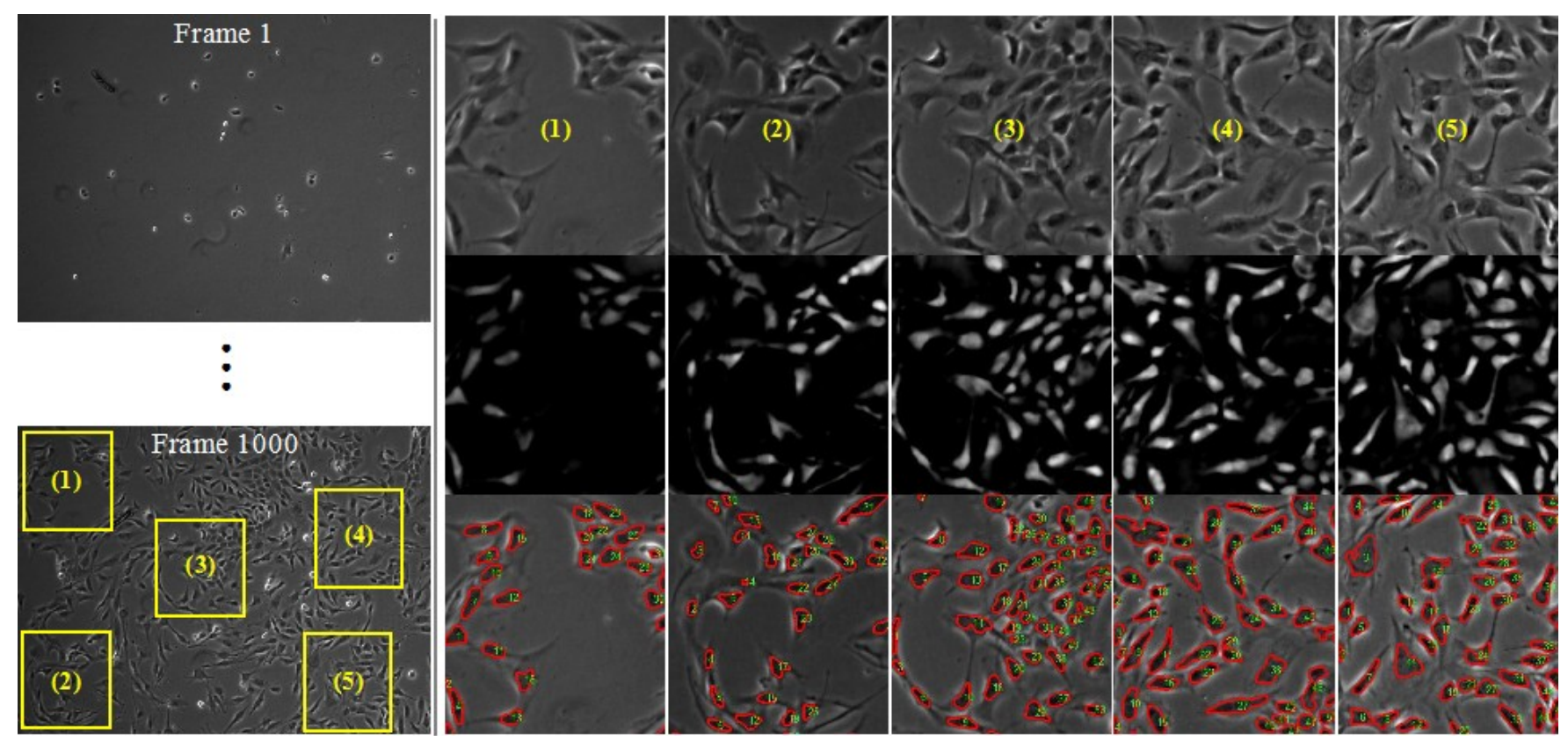

Fig. 6. Left: the first and last images of the phase contrast sequence. Right: the zoomed-in details of input phase contrast images (top row), restored phase contrast images (middle row), and segmentation by thresholding the restored images (bottom row where cells are labelled by red boundaries and green IDs).

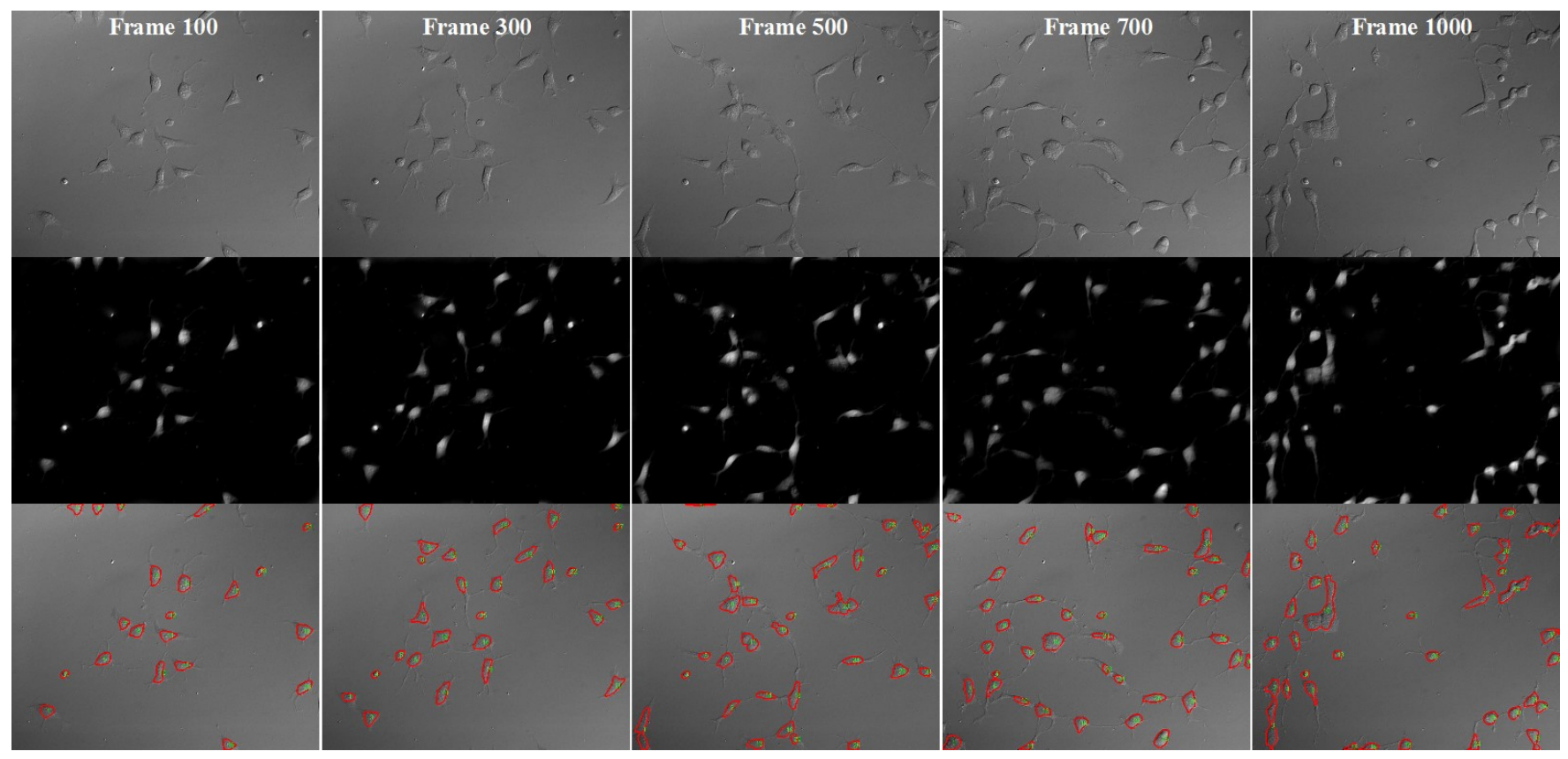

Fig. 7. Top row: input DIC images; Middle row: restored DIC images; Third row: segmentation by thresholding the restored images (cells are labelled by red boundaries and green IDs). 